\title{
Escala Brasileira de Coping para Adolescentes (EBCA): versão revisada
}

\author{
Brazilian Coping Scale for Adolescents (EBCA): revised version
}

\author{
Sheila Gonçalves Câmara ${ }^{\mathrm{I}}$ \\ Mary Sandra Carlotto ${ }^{\text {II }}$
}

\begin{abstract}
Resumo
Este artigo objetivou revisar a Escala Brasileira de Coping para Adolescentes (EBCA) considerando o modelo teórico de coping de duas dimensōes, foco no problema e foco na emoção. A versão revisada constitui-se de 31 itense foi aplicada em 802 adolescentes de escolas públicas de uma cidade da região metropolitana de Porto Alegre. Os dados foram submetidos à análise fatorial confirmatória que indicou adequados índices de ajustes e alfas satisfatórios para um modelo composto por 15 itens, 8 que avaliam as estratégias com foco na emoção e 7 com foco no problema. O uso da EBCA-Revisada pode contribuir tanto para o quanto para intervençôes com vistas ao incremento de estratégias que contribuam para uma maior qualidade de vida na adolescência.
\end{abstract}

Palavras-chave: adolescência; coping; escala; análise fatorial.

\begin{abstract}
This article aimed to review the Brazilian Coping Scale for Adolescents (EBCA) - Considering the theoretical model of two dimensions coping, focus on the problem and focus on emotion. The revised version consists of 31 items and was applied to 802 adolescents from 12 public schools in a city in metropolitan area of Porto Alegre. Data were analysed using confirmatory factor analysis, witch indicated satisfactory indicators of adjustment and suitable alpha for a model composed by 15 items, 8 assessing the strategies focused on emotion and 7 focusing on the problem. The use of EBCA can contribute to both the diagnosis and interventions aiming to increase strategies that contribute to a higher quality of life in adolescence.
\end{abstract}

Key-words: adolescents; coping; factor analysis.

${ }^{\mathrm{I}}$ Docente do Curso de Psicologia e do PPG em Psicologia e Saúde da Fundação Universidade Federal de Ciências da Saúde de Porto Alegre (UFCSPA).

II Docente do Curso de Psicologia e do PPG em Psicologia da Universidade do Vale do Rio dos Sinos (UNISINOS).

Estratégias de enfrentamento ao estresse entre adolescentes são identificadas como os recursos utilizados para lidar adequadamente com situaçôes que envolvem mudanças ou um estressor que o adolescente avalia como incontrolável (Hudson, Moffett, \& McCabe, 2015). A adolescência é um período marcado por desafios constantes referentes a eventos estressores reais ou imaginários (Caires \& Silva, 2011). As formas de lidar com o estresse estão sendo desenvolvidas e testadas. A etapa é vinculada a uma grande variedade de mudanças biológicas, cognitivas e de experimentaçáo social, entre outras (Guerreiro, Cruz, Figueira, \& Sampaio, 2014).

O adolescente está, portanto, reestruturando sua identidade frente a esta série de mudanças vitais, as quais representam estressores diferentes daqueles presentes na infância. Além disso, espera-se do adolescente a representaçáo de papéis aceitos socialmente e uma adequação massiva a normas e padróes de comportamento (Câmara \& Carlotto, 2007). Segundo os autores, nesse contexto, os adolescentes testam diferentes capacidades para lidar com eventos estressores. A forma como esses eventos são vivenciados impactam no seu desenvolvimento tornando-se fundamental compreender as dificuldades encontradas nesse processo. Esse período do ciclo vital, considerado pela Organização Mundial de Saúde (WHO, 1995) como a faixa etária entre os 10 e os 19 anos, portanto, precisa ser considerado especificamente, quando se trata da utilização de estratégias de coping.

Os estudos sobre coping derivaram das pesquisas sobre estresse (Folkman \& Lazarus, 1980). O estresse é entendido como o resultado de uma avaliação que o indivíduo faz do desequilíbrio entre seus recursos/capacidades e as demandas da situação. As estratégias que o sujeito leva em conta nesta avaliação são considerados recursos de coping (Schaefer, Coyne, \& Lazarus, 1981).

De acordo com Compas et al. (2001), entretanto, o modelo de Folkman e Lazarus (1984), assim como outros elaborados nos anos 80, são modelos desenvolvidos para adultos e, com isso, desconsideram o aspecto desenvolvimental que é fundamental para a população de crianças e adolescentes. Nesse sentido, é importante avaliar o quanto os aspectos desenvolvimentais estáo presentes nas conceitualizaçóes do processo de coping, pois isso vai estabelecer o escopo do que deve e pode ser incluído nas suas medidas de avaliação para crianças e adolescentes (Lindsay et al., 2015).

No modelo de Compas et al. (2001) coping é definido como "esforços voluntários conscientes para 
regular emoção, cognição, comportamento, fisiologia e o ambiente em resposta a eventos ou circunstâncias estressantes. Estes processos regulatórios descrevem e são limitados pelo desenvolvimento biológico, cognitivo, social e emocional do indivíduo. O nível de desenvolvimento do indivíduo tanto contribui para os recursos que estão disponíveis para o enfrentamento quanto limita os tipos de resposta de enfrentamento que o indivíduo pode apresentar" (p. 89).

Uma abordagem que tem sido amplamente utilizada em diversos modelos de coping refere-se à forma de lidar com as situaçóes estressantes, que pode ser orientada à resoluçáo do problema ou voltada para o manejo ou redução das emoçóes associadas à situação estressante (Carver, Scheier, \& Weintraub, 1989). O coping centrado no problema é utilizado para controlar a discrepância entre a pessoa e a circunstância estressora, via solução de problemas e tomada de decisão; o coping centrado na emoçáo tem como objetivo controlar a emoção resultante do estresse (Diniz \& Zanini, 2010; Veloso-Besio et al., 2010).

No que se refere à mensuração de coping, é preciso considerar a difícil delimitação conceitual, uma vez que conceitos e definiçóes têm sido estabelecidos juntamente com a elaboração de instrumentos de medida. Ayers, Sandler e Twohey (1998) estabeleceram alguns critérios para a elaboraçáo de escalas de coping entre crianças e adolescentes, apresentando vantagens e desvantagens das estratégias utilizadas. Os autores defendem que os instrumentos de coping devem ser desenvolvidos a partir de um modelo teórico que estabeleça, a priori, as dimensóes em estudo. Embora essa estratégia melhore a validade externa no instrumento em detrimento da interna, torna-se mais plausível a replicabilidade dos resultados para diferentes populaçóes. Entre o desenho do instrumento baseado unicamente na teoria, na análise fatorial exploratória ou na confirmatória, os autores defendem o uso da análise fatorial confirmatória, uma vez que esta avalia o modelo teórico e não está sujeita às inconsistências internas do construto.

Em relação à população adolescente, estudo de revisão da literatura realizado por Compas et al. (2001) identificou todas as escalas criadas entre 1988 e 1999, tendo chegado ao número de 20 instrumentos. Em 2010, estudo de revisão conduzido por García (2010), com publicaçôes em língua inglesa, contemplando o período entre 1998 e 2009, revelou 48 estudos com mensuração de coping entre crianças e adolescentes.

No Brasil, foram identificados apenas três instrumento construídos ou adaptados para adolescentes. $\mathrm{O}$ primeiro é o Inventário Multifatorial de Coping para
Adolescentes (IMCA-43), desenvolvido por Antoniazzi (2000), seguindo o modelo Aproximação e Evitação. Estudo sobre a fidedignidade e validade de construto do instrumento de 43 itens identificou quatro fatores: açóes diretas, apoio social, negação e autocontrole, todos com boa consistência interna (Balbinotti, Barbosa, \& Wiethaeuper, 2006).

O segundo é a Adolescent Coping Strategies (ACS), com 79 itens fechados agrupados em 18 estratégias e um item aberto para contemplar estratégias não abordadas no instrumento. Conceitualmente, as estratégias estão divididas em três estilos de coping: focado no problema, referência a outros e coping não produtivo (Frydenberg \& Lewis, 1997). Em estudo brasileiro, a ACS apresentou oito fatores com índices satisfatórios de consistência interna. (Câmara, Sarriera, \& Remor, 2002).

O terceiro é a Escala Brasileira de Coping para Adolescentes (EBCA), de Câmara e Carlotto (2009). Essa foi construída a partir de estudos qualitativos e revisão da literatura e na sua versão final constituiu-se de 23 itens distribuídos em cinco dimensôes: evitação, apoio social, apoio profissional, coping ativo e religiosidade, que apresentam adequadas propriedades psicométricas. De acordo com Carver, Scheier e Weintraub (1989), apoio social, apoio profissional e coping ativo são consideradas estratégias com foco no problema e as de religiosidade e evitaçáo como de foco na emoçáo. Nesse sentido, a escala contempla mais estratégias focalizadas no problema do que na emoçáo, o que pode ser considerado um aspecto a ser revisado.

Estudos demonstram a importância das estratégias de coping utilizadas pelos adolescentes para sua adaptação social e como medida de manutenção e promoção da saúde física e psíquica (Diniz \& Zanini, 2010; Serafini \& Bandeira, 2011). Conhecer quais as estratégias utilizadas pelos adolescentes deve constituir uma preocupação no âmbito da psicologia (Veloso-Besio et al., 2010), por terem ligação íntima com adaptação social, interferindo na saúde física e mental (Caires \& Silva, 2011). Trata-se de um momento fundamental para desenvolver intervençóes que estimulem capacidades de coping para uma vida mais saudável e gratificante (Guerreiro et al., 2014).

Considerando a carência de instrumentos destinados a adolescentes, especialmente que contemplem um modelo desenvolvimental, este estudo buscou, a partir do modelo de Compas et al. (2001), e da abordagem de foco no problema e foco na emoção, de Carver, Scheier e Weintraub (1989), revisar a Escala Brasileira de Coping para Adolescentes (EBCA) de Câmara e Carlotto (2009). 


\section{MÉTODO}

\section{Participantes}

Os adolescentes foram selecionados por acessibilidade em 12 escolas públicas estaduais de uma cidade de grande porte da regiáo metropolitana de Porto Alegre, RS. O instrumento foi aplicado em 846 adolescentes estudantes de sexto e sétimo ano do ensino fundamental. Desses, foram excluídos 39 por não haverem preenchido o mínimo de $90 \%$ da escala e cinco por representarem casos extremos (outliers), detectados por meio do D2 de Mahalanobis. Assim, a amostra final constitui-se de 802 participantes. Quanto às características demográficas, a maioria dos participantes identificou-se como do sexo feminino $(60,2 \%)$ e a idade variou de 11 a 19 anos $(M=12,6 ; D P=1,40)$.

\section{Instrumento}

Escala Brasileira de Coping para Adolescentes (EBCA), versão revisada, baseada na EBCA de Câmara e Carlotto (2009). A escala original constitui-se de 23 itens distribuídos em cinco dimensôes: apoio social (5 itens; $\alpha=0,75$ ), apoio profissional (3 itens; $\alpha=0,81$ ), coping ativo ( 5 itens; $\alpha=0,67$ ), religiosidade ( 3 itens; $\alpha=0,65$ ), e evitação (7 itens; $\alpha=0,75$ ). Os itens são avaliados por meio de um escala tipo Likert de cinco pontos, avaliando frequência de utilização das estratégias ( 0 - nunca, 1 - poucas vezes, 2 - algumas vezes, 3 - muitas vezes e 4 - sempre).

Para atender à abordagem de foco no problema e foco na emoção (Carver, Scheier, \& Weintraub, 1989), nove itens foram adicionados, visando fundamentalmente complementar a dimensão de estratégias com foco na emoçáo uma vez que essas se apresentavam em menor diversidade em comparação às estratégias com foco no problema, totalizando 32 itens.

\section{Construção de novos itens}

Os itens foram construídos de acordo com os princípios e técnicas da construção de testes psicométricos (Pasquali, 2010). A metodologia seguiu as seguintes etapas de desenvolvimento metodológico de itens: a) revisão da literatura, com operacionalização de modelo teórico sobre coping entre adolescentes; e, b) consulta a instrumentos de avaliação de coping como Inventário Multifatorial de Coping para Adolescentes (IMCA-43), desenvolvido por Antoniazzi (2000), Adolescent Coping Strategies (ACS) de (Frydenberg \& Lewis, 1997) e COPE de Carver, Scheier e Weintraub (1989).

Para verificar a compreensão e a relevância dos itens para as estratégias de coping utilizadas por adolescentes, focadas no problema e na emoção, a versáo inicial de 32 itens foi enviada a três psicólogos, experts nos temas de coping e/ou adolescência juntamente com um protocolo estandartizado de avaliação no qual deveriam: a) avaliar o aspecto semântico dos itens (clareza dos conteúdos e compreensibilidade dos termos para adolescentes de diferentes classes sociais); b) analisar os itens e classificá-los em três categorias (adequados expressam opinião adequada sobre o tema, plausiveis frase não totalmente adequada, mas que expresse algum aspecto do construto, e ingênuos - nem adequado e nem plausível); c) categorizar os itens de acordo com os fatores que representavam as estratégias de coping focadas no problema e focadas na emoçáo; e, d) se o item deveria ser aceito tal como estava, revisado ou eliminado.

As respostas dos experts foram avaliadas através de porcentagens para modificação e exclusão de itens. O critério estabelecido para a tomada de decisão acerca da manutenção, modificação ou rejeição de itens foi: a) para compreensibilidade, o item foi revisado quando o acordo acerca da clareza foi menor que $80 \%$, b) relevância para o construto - $o$ item foi retirado quando menos de $80 \%$ dos juízes o considerassem relevante para o tema, c) para modificação, o item foi reescrito quando mais de $20 \%$ dos experts recomendaram modificação, e d) quanto à correspondência item-dimensão, o item foi mantidos quando houve acordo entre mais de $80 \%$ dos experts.Foram revisados 4 itens e 3 foram eliminados, restando 29.

\section{Procedimentos de coleta dos dados}

A aplicação inicial do instrumento foi acordada previamente com a direção de cada escola. Foram combinados três retornos semanais para captar os alunos faltantes no dia das coletas anteriores. Após a autorização, a coleta de dados foi realizada de forma grupal, em salas de aula, após o turno escolar, por bolsistas de iniciação científica e acadêmicos de psicologia treinados para a atividade.

O tempo médio de aplicação foi de 10 minutos. Os participantes responderam ao instrumento de pesquisa mediante assentimento dos estudantes e autorizaçáo dos responsáveis (assinatura do Termo de Consentimento Livre e Esclarecido), no caso dos estudantes menores de 18 anos. O projeto de pesquisa foi 
aprovado pelo Comitê de Ética em Pesquisa da instituição de afiliação da primeira autora (protocolo: 2012-130.381).

\section{Procedimentos para análise dos dados}

Primeiramente realizaram-se análises descritivas de caráter exploratório a fim de avaliar, no banco de dados, a distribuição dos itens, casos omissos e identificação de extremos por meio do $\mathrm{PASW}^{\odot}$ version 17 (SPSS/PASW, Inc., Chicago, IL). A análise fatorial confirmatória foi realizada por meio do Amos Graphics versão18, pelo método de máxima verossimilhança.

\section{RESULTADOS}

\section{Estatística descritiva}

Foram avaliadas as médias dos 29 ítens, que variaram de 0,62 a 2,66, com desvios padrão entre $0,50 \mathrm{e}$ 1,30 , indicando homogeneidade. As correlaçóes itemtotal foram: foco no problema (entre $r=0,14$ e $r=0,59$ ) e foco na emoção $(r=0,42$ e entre $r=0,53)$, mostrando-se satisfatórias. Uma vez avaliados estes requisitos, procedeu-se o cálculo dos coeficientes alfa de Cronbach, que variaram de 0,71 a 0,81 (Tabela 1 ).

Identifica-se que as médias mais elevadas foram as obtidas pelos itens que formam a dimensão Foco

\section{Tabela 1}

Estatística Descritiva e Consistência Interna dos Itens e Dimensóes da Escala Brasileira de Coping para Adolescentes ( $n=719)$.

\begin{tabular}{|c|c|c|c|c|}
\hline Subescala/Item & $M(\mathrm{DP})$ & $\begin{array}{l}\text { Correlaçáo } \\
\text { item-total }\end{array}$ & Ass & $\begin{array}{c}\text { Alfa } \\
\text { sem item }\end{array}$ \\
\hline Foco no Problema $(\alpha=0,81)$ & $2,35(1,28)$ & & & \\
\hline 1.Procuro um amigo de confiança... & $1,68(1,27)$ & 0,35 & $-0,32$ & 0,81 \\
\hline 6.Procuro pessoas competentes no tema... & $1,27(1,28)$ & 0,54 & 0,28 & 0,79 \\
\hline 4.Peço ajuda a profissionais... & $2,64(0,99)$ & 0,47 & 0,65 & 0,80 \\
\hline 10.Penso em maneiras possíveis para enfrentar ... & $2,42(1,25)$ & 0,43 & $-0,51$ & 0,80 \\
\hline 11.Procuro o apoio de meus pais e amigos & $2,68(1,00)$ & 0,59 & $-0,35$ & 0,79 \\
\hline 12.Procuro as causas do problema ... & $1,95(1,16)$ & 0,32 & 1,00 & 0,81 \\
\hline 14.Procuro outras pessoas para conversar... & $2,09(1,09)$ & 0,55 & 0,09 & 0,79 \\
\hline 16.Procuro saber como outras pessoas resolveriam o problema & $2,30(1,12)$ & 0,37 & 0,02 & 0,81 \\
\hline 17.Uso todas as minhas capacidades... & $1,74(1,16)$ & 0,39 & $-0,24$ & 0,81 \\
\hline 18.Busco uma forma de ação ... & $2,66(0,99)$ & 0,40 & 0,04 & 0,81 \\
\hline 19.Paro para pensar em como estou agindo... & $2,11(1,12)$ & 0,40 & $-0,40$ & 0,81 \\
\hline 21.Procuro conselho e/ou ajuda... & $1,54(1,26)$ & 0,53 & $-0,05$ & 0,80 \\
\hline 22.Busco o conselho de profissionais & $2,35(1,28)$ & 0,51 & 0,40 & 0,80 \\
\hline Foco na Emoção $(\alpha=0,73)$ & $1,29(0,50)$ & & & \\
\hline 2.Tento esquecer o problema & $1,00(1,02)$ & 0,29 & 0,82 & 0,72 \\
\hline 3.Entrego os problemas para que uma força superior... & $1,39(1,31)$ & 0,28 & 0,64 & 0,73 \\
\hline 5.Tento ignorar o problema & $0,71(0,95)$ & 0,40 & 0,82 & 0,72 \\
\hline 7.Desisto de tentar resolver o problema & $0,56(0,89)$ & 0,38 & 0,93 & 0,72 \\
\hline 8. Tento evitar o problema... & $0,91(1,06)$ & 0,36 & 0,87 & 0,72 \\
\hline 13.Peço a uma força superior que me proteja... & $2,63(1,30)$ & 0,14 & $-0,54$ & 0,74 \\
\hline 15.Tento não pensar e entro em redes sociais... & $0,67(0,93)$ & 0,40 & 0,83 & 0,72 \\
\hline 20.Espero que o problema se resolva sozinho & $0,62(0,91)$ & 0,43 & 0,86 & 0,72 \\
\hline 23.Não faço nada... & $0,88(0,98)$ & 0,33 & $-0,88$ & 0,72 \\
\hline 24. Sinto-me abalado emocionalmente e choro & $1,43(1,22)$ & 0,41 & $-0,48$ & 0,71 \\
\hline 25.Chateio-me e transfiro esse sentimento para outras pessoas & $0,70(0,93)$ & 0,43 & 0,90 & 0,72 \\
\hline 27.Sou tomado(a) por sentimentos negativos & $1,60(1,12)$ & 0,42 & 0,34 & 0,71 \\
\hline 28. Tenho esperanças que a situação se resolva... & $2,54(1,17)$ & 0,17 & $-0,45$ & 0,74 \\
\hline 29. Começo a sentir dores no meu corpo & $1,60(1,28)$ & 0,29 & $-0,42$ & 0,73 \\
\hline 30.Uso algum tipo de substância química & $1,23(1,35)$ & 0,39 & $-0,70$ & 0,72 \\
\hline 31.Durmo e/ou como mais do que o habitual & $2,16(1,30)$ & 0,30 & $-0,11$ & 0,73 \\
\hline
\end{tabular}


no Problema (itens 11, 18 e 4) e as mais baixas correspondem aos que compóem a dimensão Foco na Emoção (itens 7, 20e 15).A maioria dos itens da correlação item-total corrigida alcançou valores superiores a 0,30 , com exceçáo dos itens $2,3,13,18$ e 29. Todos os itens contribuíram para o aumento da consistência interna, avaliada pelo alfa de Cronbach da subescala a qual pertenciam.

\section{Análise fatorial confirmatória}

O modelo de dois fatores foi testado por meio de análise fatorial confirmatória, indicando ajuste adequado à teoria subjacente. Nesse modelo foram eliminados 4 itens, devido a sua baixa carga fatorial (inferior a $0,30)$. Também foram eliminados 10 itens, devido à elevada correlaçáo com itens de seu mesmo fator (Foco na Emoção). O modelo alcançou índices adequados para a amostra de acordo com Byrne (1998) $\left(\chi^{2}=257,18\right.$, $p \leq 0,001 ; g l=89 ; \chi^{2} / g l=2,89 ; \mathrm{GFI}=0,96 ; \mathrm{RMSEA}=0,04$ $[\mathrm{IC}=0,04-0,05] ; \mathrm{CFI}=0,92 ; \mathrm{PNFI}=0,74)$.

Devido à prova $\chi^{2}$ ser sensível ao tamanho da amostra, foi calculada a razão entre seu valor e os graus de liberdade. Embora não exista um valor crítico exato para decidir sobre a adequação ou náo do modelo, na prática se aceitam índices que sejam iguais ou inferiores a 5,00 (Byrne, 1998). Portanto, o valor obtido neste estudo indica que o modelo se ajusta aos dados $\left(\chi^{2} / g l=2,89\right)$. $O$ modelo final encontra-se exposto na Figura 1. A EBCA, versão revisada, encontra-se em anexo.

A confiabilidade do modelo final foi avaliada pelo índice alfa de Cronbach. O fator 1 (Foco no problema) alcançou um alfa de 0,74 e fator 2 (Foco na Emoçáo) obteve alfa de 0,76 .

\section{DISCUSSÁO}

O presente estudo teve como objetivo revisar a Escala Brasileira de Coping para Adolescentes (EBCA), de Câmara e Carlotto (2009), a partir do modelo teórico de Compas et al. (2001), e da abordagem de foco no problema e foco na emoção, de Carver, Scheier e Weintraub (1989). Os resultados de adequaçáo e propriedades psicométricas permitem afirmar que o modelo final obtido está adequado para a avaliação de estratégias de coping entre adolescentes escolares.

Verifica-se que as estratégias com foco no problema tendem a emergir como uma dimensão bem definida nos modelos de coping entre crianças e adolescentes. No presente estudo esta dimensão adquiriu uma conotação de busca por apoio social e instrumental, no

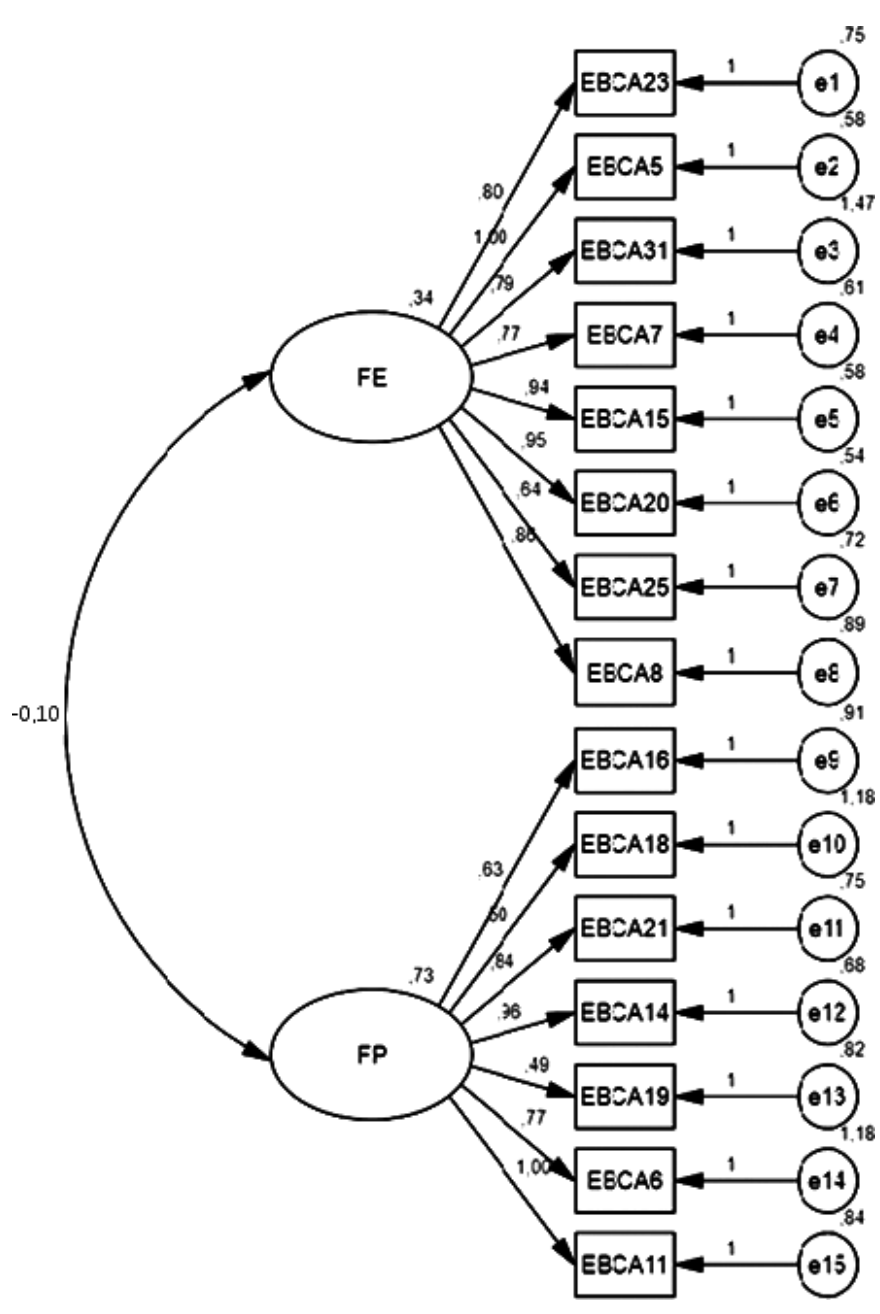

Figura 1.

\section{Resultados do modelo fatorial hipotetizado para a}

"Escala Brasileira de Coping para Adolescentes

sentido da busca por informaçóes ou assistência. No estudo de Brodzinsky et al. (1992), abusca de apoio surge como uma estratégia para a resoluçáo de problemas interpessoais. Saha (2015) verificou a importância do apoio social para a satisfaçáo com a vida em adolescentes.

Tais estratégias caracterizam-se, também, por atividade cognitiva como forma de aproximação ao problema, com alguma açáo direta no sentido de amenizar as circunstâncias ou resolvê-las. A definição da categoria presente na EBCA é, portanto, bastante similar à de Lazaruse Folkman (1984). No entanto, caracterizase, ainda, por um espaço de reflexão sobre as próprias açôes, o que a aproxima da definição de Carver, Scheier e Weintraub (1989). No estudo de Ayers et al. (1996) os autores encontraram como componentes os aspectos de tomada de decisão, solução direta de problemas, busca por entendimento e reestruturação positiva.

As estratégias com foco na emoção apresentam conteúdos de evitação e certa busca por formas de afastamento do problema, bem como um item de caráter 
emocionalmente aversivo com a transferência de emoçôes para outras pessoas. A evitaçáo implica em esforços para náo se defrontar com o problema ou esperar que ele se resolva sozinho. Essa dimensão aproxima-se à fuga e esquiva, proposta por Lazarus (Folkman\&Lazarus, 1985) e também à dimensão de negação de Carver et al. (1989).

A construção da EBCA tentou suprir a lacuna atual de instrumentos breves validados que avaliem estratégias de coping em adolescentes. Assim, dos 31 itens iniciais da escala revisada, esperava-se um modelo parcimonioso, que contemplasse as possibilidades e limites da etapa do desenvolvimento da adolescência. A configuração do modelo final retrata comportamentos característicos desse período com relação às estratégias com foco no problema. $\mathrm{Na}$ adolescência a busca por informaçáo e apoio de pares, professores e familiares representa comportamentos prototípicos na busca de satisfação com a vida e desenvolvimento em geral (Saha et al., 2014). Por outro lado, desligar-se e evitar problemas também se constitui em comportamentos habituais nesta etapa, a fim de evitar emoçôes negativas e aumentar as positivas (Chinazzo, Câmara, \& Frantz, 2014). Os valores da correlação item-total corrigida foram elevados, evidenciando que cada dimensão da EBCA-revisada pode ser considerada com uma função linear dos itens que a compóem. Os valores obtidos para assimetria indicam boa adequação dos dados. Os valores mais elevados foram identificados nos itens 7 $(A s=0,93)$ e $25(A s=0,90)$, pertencentes à dimensão de foco na emoçáo, e $12(A s=1,00)$, que pertence à dimensão de foco no problema. Considera-se de esses valores náo comprometem a normalidade. De acordo com Miles e Shevlin (2005), os valores de assimetria aceitáveis estão contidos no intervalo de \pm 1 .

Os índices de fidedignidade dos fatores foram satisfatórios, superiores a 0,70 . Com esses coeficientes, é possível utilizar, integramente, a escala tanto quanto os fatores, independentemente, para a avaliaçáo de estratégias de coping em adolescentes.

Neste estudo foi avaliada a versão original da EBCA (Câmara \& Carlotto, 2009) e a análise fatorial confirmatória corroborou a estrutura teórica de dois fatores, conforme a abordagem de foco no problema e foco na emoção (Carver, Scheier, \& Weintraub, 1989). Verificou-se que os fatores apresentam relação negativa, embora fraca, entre si $(r=-0,10 ; p \leq 0,05)$. Todos os itens apresentaram carga fatorial significativa, variando de $\lambda=0,35$ a $\lambda=0,70$. $O$ menor valor foi obtido pelos itens $31(\lambda=0,35)$ e $18(\lambda=0,36)$.

$\mathrm{O}$ presente estudo indica que os diversos aspectos de validade do instrumento apresentaram resultados satisfatórios. Esses dados evidenciam que que a EBCArevisada possui propriedades psicométricas adequadas para o estudo das estratégias de coping em adolescentes escolares brasileiros.

Uma limitação do presente estudo diz respeito à população na qual a escala foi avaliada. Há uma especificidade em termos amostrais, uma vez que a validação do instrumento ocorreu em um município do sul do Brasil, com adolescentes escolares. Nesse sentido, seriam importantes novos estudos com adolescentes de outras regiôes brasileiras, bem como com adolescentes do sistema privado de ensino, ou que não se encontrem no contexto escolar.

Quanto aos dados psicométricos, a sugestão é que sejam conduzidos novos estudos com a EBCA utilizando outras formas de avaliação da fidedignidade, como é o caso do teste-resteste para avaliar a estabilidade do instrumento. Estudos longitudinais que contemplassem as diferenças de gênero ou faixas etárias (dentro da adolescência) também contribuiriam para sua utilização de forma mais ampla.

Estudos futuros também devem explorar a trajetória de desenvolvimento das estratégias de enfrentamento, especialmente no contexto de estressores específicos. Tais pesquisas são importantes para ampliar a compreensão do desenvolvimento das habilidades de enfrentamento, bem como a forma como surgem os diversos perfis de enfrentamento e suas consequências em longo prazo (Lindsay et al., 2015).

Contemplar as especificidades da etapa do desenvolvimento, coadunadas com estudos psicométricos adequados, pode tornar a EBCA-Revisada uma escala de importante utilizaçáo. Acredita-se que o uso da escala para a avaliação das estratégias de coping de adolescentes pode contribuir tanto para o diagnóstico das populaçóes em estudo, quanto para intervençóes com vistas ao incremento de estratégias que contribuam para uma maior qualidade de vida na adolescência e na vida adulta.

\section{REFERÊNCIAS}

Antoniazzi, A. S. (2000). Desenvolvimento de Instrumentos para a Avaliação de Coping em Adolescentes Brasileiros. [Tese de Doutorado, nãopublicada], Universidade Federal do Rio Grande do Sul, Instituto de Psicologia, Curso de Pós-Graduação em Psicologia do Desenvolvimento. Porto Alegre.

Ayers, T. S., Sandler, I. N., \& Twohey, J. (1998). Conceptualization and measurement of coping in children and adolescents. Em T. H. Ollendick \& R. 
J. Prinz (Eds.), Advances in clinical child psychology (pp.243-301). New York: Plenum Press.

Balbinotti, M. A. A., Barbosa, M. L. L., \& Wiethaeuper, D. (2006). Consistência interna e fatorial do Inventário Multifatorial de Coping para Adolescentes. PsicoUSF, 11(2), 175-183.

Brodzinsky, D. M., Elias, M. J., Steiger, C., \& Simon, J. (1992). Coping scale for children and youth: Scale development and validation. Journal of Applied Developmental Psychology, 12(2), 195-214. doi. org/10.1016/0193-3973(92)90029-H

Byrne, B. M. (1998). Structural Equation Modeling with LISREL, PRELIS and SIMPLIS: basic concepts, applications and programming. Mahwah, New Jersey: Lawrence Erlbaum Associates.

Caires, S., \& Silva, C. (2011). Fatores de stress e estratégias de coping entre adolescentes do $12^{\circ}$ ano de escolaridade. Estudos de Psicologia (Campinas), 28(3), 295-306. doi.org/10.1590/ S0103-166X2011000300001

Câmara, S. G., \& Carlotto, M. S. (2007). Coping e gênero em adolescentes. Psicologia em Estudo, 12(1), 87-93. doi.org/10.1590/S1413-73722007000100011

Câmara, S. G., Sarriera, J. C., \& Carlotto, M. S. (2007). Fatores associados a condutas de enfrentamento violento entre adolescentes escolares. Estudos de Psicologia (Natal), 12(3), 213-219.

Câmara, S. G., \& Carlotto, M. S. (2009). Construção e validação de uma escala para avaliar coping em adolescentes. I Congreso Internacional de Investigación y Práctica Profesional en Psicología XVI Jornadas de Investigación Quinto Encuentro de Investigadores en Psicología del MERCOSUR. Facultad de Psicología - Universidad de Buenos Aires, Buenos Aires. p.500-501.

Câmara, S. G., Sarriera, J. C., \& Remor, E. A. (2002). Análise fatorial da escala de afrontamento para adolescentes (ACS) em uma amostra de jovens de Porto Alegre. Aletheia, 16, 15-32.

Carver, C. S., \& Connor-Smith, J. K. (2010). Personality and coping. Annual Review of Psychology, 61, 79-704. dx.doi.org/10.1146/annurev.psych.093008.100352

Carver, C. S., \& Vargas, S. (2011). Stress, coping and health. Em H. S. Friedman (Ed.), The Oxford Handbook of Health Psychology (pp. 162-188). New York: Oxford University Press.

Carver, C. S., Scheier, M. F., \& Weintraub, J. K. (1989). Assessing coping strategies: A theoretically based approach. Journal of Personality and Social Psychology, 56(2), 267-283. doi. org/10.1037/0022-3514.56.2.267
Chinazzo, I. R., Câmara, S. G., \& Frantz, D. G. (2014). Comportamento sexual de risco em jovens: aspectos cognitivos e emocionais. Psico-USF, 19(1), 1-12. doi:10.1590/S1413-82712014000100002

Compas, B. E., Connor-Smith, J. K., Saltzman, H., Thomsen, A. H., \& Wadsworth, M. E. (2001). Coping with stress during childhood and adolescence: problems, progress, and potential in theory and research. Psychological Bulletin, 127(1), 87-127. doi.org/10.1037/0033-2909.127.1.87

Diniz, S. S., \& Zanini, D. S. (2010). Relação entre fatores de personalidade e estratégias decopingem adolescentes. Psico-USF, 15(1), 71-80.doi. org/10.1590/S1413-82712010000100008.

Folkman, S., \& Moskowitz, J. T. (2004). Coping: Pitfalls and promise. Annual Review of Psychology, 55, 745774. doi.org/10.1037/0022-3514.46.4.839

Folkman, S., \& Lazarus, R. S. (1980). An analysis of coping in a middle-aged community sample.Journal of Health and Social Behavior, 21(3), 219-239. doi. org/10.2307/2136617

Folkman, S., \& Lazarus, R.S. (1984). If it changes it must be a process: study of emotion and coping during three stages of a college examination. Journal of Personality and Social Psychology, 48(1),150-170. doi.org/10.1037/0022-3514.48.1.150

Folkman, S., Schaefer, C., \& Lazarus, R.S. (1979). Cognitive processes as mediators of stress and coping. Em V. Hamilton \& D. M. Warburton (Eds.), Human stress and cognition: An informationprocessing approach (pp. 265-298). London: Wiley.

Frydenberg, E., \& Lewis, R. (1997). Escalas de afrontamiento para adolescentes: Manual. Madrid: Publicaciones de Psicología Aplicada.

Garcia, C. (2010). Conceptualization and measurement of coping during adolescence: A review of the literature. Journalof Nursery Scholarship, 42(2), 166185. doi.org/10.1111/j.1547-5069.2009.01327.x

Guerreiro, D. F., Cruz, D., Figueira, M. L., \& Sampaio, D. (2014). Estudo de adaptaçáo e características psicométricas da versão portuguesa da Adolescent Coping Scale - Escala de Coping para Adolescentes. Acta Médica Portuguesa,27(2), 166-180.

Hudson, T. M., Moffett, N. L., \&McCabe, K. (2015). What is the Impact of Stress-Coping Skills on Levels of Motivation in Adolescents? Georgia Educational Researcher,12(1), Available at: http:// digitalcommons.georgiasouthern.edu/gerjournal/ vol $12 /$ iss $1 / 2$

Lazarus, R. S, (1991). Emotion and adaptation. New York: Oxford University Press. 
Lindsay, D. E. \&Kouros, C., Frankel, S.A., McCauley, E., Diamond, G., Kelly A.,...\& Garber, J. (2015). Longitudinal relations between stress and depressive symptoms in youth: coping as a mediator. Journal of Abnormal Child Psychology, 43, 355-368.doi: 10.1007/s10802-014-9906-5

Miles, J., \& Shevlin, M. (2001). Applying regression and correlation: A guide for students and researchers. London: Sage Publications.

Ott, R. L., \& Longnecker, M. (2001). An introduction to statistical methods and data analysis (Fifth Edition). Pacific Grove, CA: Duxbury.

Pasquali, L. (2010). Instrumentalização psicológica: fundamentos e práticas. Porto Alegre: Artmed.

Roth, S., \& Cohen, L. J. (1986). Approach, avoidance, and coping with stress. American Psychologist, 41(7), 813-819.doi.org/10.1037/0003-066X.41.7.813

Sahaa, R., Huebner, R. S., Hills, K. L., Malone, P. S., \& Valois, R. F. (2014). Social Coping and Life Satisfaction in Adolescents. Social Indicators Research, 115, 241-252. doi: 10.1007/ s11205-012-0217-3

Schaefer, C., Coyne, J. C., \& Lazarus, R. S. (1981).The health-related functions of social support.Journal of Behavioral Medicine, 4, 381-406. doi.org/10.1007/ BF00846149
Serafini, A. J., \& Bandeira, D. R. (2011). A influência da rede de relaçóes, do coping e do neuroticismo na satisfação de vida de jovens estudantes. Estudos de Psicologia, 28(1), 15-25. doi.org/10.1590/ S0103-166X2011000100002

Veloso-Besio, C., Caqueo-Arancibia, W., CaqueoUrizar, A., Muñoz-Sánchez, Z., \&VillegasAbarzúa, F. (2010). Estrategias de afrontamiento en adolescentes. Revista de Psicología,22(1), 23-34. doi.org/10.1590/S1984-02922010000100003

WHO (1995). The use and interpretation of anthropometry. WHO Technical Report Series, 854. Geneva: WHO.

\section{Endereço para correspondência:}

E-mail: sheila.gcamara@gmail.com

E-mail: mscarlotto@pesquisador.cnpq.br

Recebido em 28/09/2015

Aceito em 15/03/2016 


\section{Anexo 1. Escala brasileira de coping para adolescentes (Versão revisada)}

Pense em um problema que você tenha tido recentemente e sua maneira de agir frente aos problemas em sua vida em geral. Indique, marcando na coluna correspondente, o quanto você pensa ou faz cada uma das alternativas. Em cada frase você deve marcar uma resposta. Não há respostas certas ou erradas. Responda de acordo com as seguintes alternativas:

$$
\begin{aligned}
& 0 \text { - Nunca } \\
& 1 \text { - Poucas vezes } \\
& 2 \text {-Algumas vezes } \\
& 3 \text { - Muitas vezes } \\
& 4 \text { - Sempre }
\end{aligned}
$$

1. Tento ignorar o problema

2. Procuro pessoas competentes no tema do problema

3. Desisto de tentar resolver o problema

4. Tento evitar o problema afastando-me dele

5. Procuro o apoio de meus pais e amigos

6. Procuro outras pessoas para conversar e verificar se podem me ajudar

7. Tento não pensar no problema e entro em redes sociais, vou ao cinema ou assisto tevê.

8. Procuro saber como outras pessoas resolveriam o problema

9. Busco sempre uma forma de ação frente ao meu problema

10. Paro para pensar em como estou agindo e por quê

11. Espero que o problema se resolva sozinho

12. Procuro conselho e/ou ajuda de algumas pessoas para resolver o problema

13. Não faço nada, pois acho que não posso resolver o problema

14. Chateio-me e transfiro esse sentimento para outras pessoas

15. Durmo e/ou como mais do que o habitual 